\title{
MOVILIDAD DE POBLACIÓN Y COMPORTAMIENTO REPRODUCTIVO: EL CASO DE BAJA CALIFORNIA
}

\author{
Por \\ Gabriel Estrella Valenzuela*
}

\begin{abstract}
RESUMEN
Este trabajo tiene como objetivo central evaluar el impacto indirecto que genera la inmigración hacia Baja California a través del comportamiento reproductivo de la población que, al migrar, ha cambiado su residencia habitual a la entidad. Para lograr ese objetivo, se analiza la información de las Encuestas Demográficas de Baja California de 1986 y 1990 con el modelo de los determinates próximos de la fecundidad. Los resultados de dicho análisis muestran, por una parte, que los patrones reproductivos de la población migrante y no-migrante son claramente diferenciados (tantoen términos de fecundidad ilegítima como de formación de uniones, de sus prácticas anticonceptivas y de lactancia postparto), y que de ello se deriva un diferencial de fecundidad que resulta ser $17.5 \%$ superior para la población migrante en 1990. Por otra parte, los resultados también permiten estimar que, por cada tres nuevos inmigrantes que por año recibe la entidad, la inmigración acumulada aporta dos nuevos residentes con los nacimientos generados por las mujeres migrantes. Dada la magnitud que adquieren esos procesos (i.e. 59 mil nuevos habitantes por año en total), se concluye sugiriendo líneas generales de acción que permitan adecuar la política de población a las condiciones específicas de Baja California. 1
\end{abstract}

\begin{abstract}
The central aim of this paper is the evaluation of the indirect impact that the process of internal migration to Baja California generates through the reproductive patterns of the population which has change its residence to the state. To attain that aim, we analyze the data from the Baja California Demographic Surveys of 1986 and 1990, applying the Bongaarts proximate determinants of fertility model. The results of that analysis show, on one hand, that the reproductive patterns of the migrant and non-migrant populations are clearly differentiated (both in terms of illegitimate fertility and of union formation, contraception prevalence, and postpartum infecundability due to lactation), and that from those patterns emerges a differential that tums out to be of $17.5 \%$ higher fertility for the migrant population. The results, on the other hand, also allow for an estimate that show that, for each 3 new migrants that arrive to the state each year, the cumulated migration generates 2 new residents of the state through the births from migrant women. Due to the magnitude of those processes (i.e. 59,000 new inhabitants a year in total), we conclude suggesting general lines of action in order to adequate population policies to the specific needs of Baja California.
\end{abstract}

* Investigador del Instituto de Investigaciones Sociales de la Universidad Autónoma de Baja California. Investigador Nacional. Becario UABC al Desempeño Académico. 


\section{INTRODUCCIÓN}

A lo largo de los últimos sesenta años de este siglo, tanto el conjunto de las seis entidades fronterizas del norte de México (i.e. Baja California, Coahuila, Chihuahua, Sonora, Nuevo León y Tamaulipas) como el de los 38 municipios de esas entidades que - por su colindancia con la frontera internacional o por la intensidad de sus interacciones con el país vecino del norte - son considerados como fronterizos, se han caracterizado por presentar un crecimiento poblacional superior al del total del país.

En relación a este proceso, Zenteno y Cruz (1992) han seffalado que - salvo en la década de 1970 a 1980 — a la vez que las seis entidades crecían más rápidamente que el país en su conjunto, de 1930 a 1990 los municipios fronterizos superaban los ritmos de crecimiento de esas seis entidades fronterizas (Zenteno y Cruz, 1992:36-37). Estos elevados ritmos de crecimiento que han caracterizado a esta zona del país han sido asociados, tanto por los autores mencionados como en otros trabajos relativos a esta área de México (e.g. CONAPO, 1988; Estrella, 1989), con los flujos de migración interna que se han dirigido durante este lapso a la zona fronteriza del norte de México, flujos que aun en el periodo de 1985 a 1990 generaron un saldo neto migratorio positivo de más de $352 \mathrm{mil}$ inmigrantes en las seis entidades fronterizas mencionadas (INEGI, 1992a:18).

Debido a la importancia que ha manifestado el crecimiento social de la población en esta zona del norte de México, dicho componente de la dinámica demográfica fronteriza ha sido evaluado en términos de su magnitud absoluta (e.g. Cabrera, 1967; Corona y Ruiz, 1982. Ordorica, 1976; Tabah y Cosío, 1970), de sus patrones de comportamiento tendencial a lo largo del tiempo (e.g. Corona, 1993; Margulis y Tuirán, 1986; Ocampo, 1981), así como de las repercusiones que genera la inmigración hacia esta área del país en materia de demandas de empleo y vivienda (e.g. CONAPO, 1988).

Es decir, que la inmigración hacia la frontera norte de México ha sido evaluada, fundamentalmente, en términos de sus impactos directos en la dinámica demográfica de esta zona. Sin embargo, recientemente se ha iniciado el análisis de lo que podemos denominar el impacto indirecto de la inmigración sobre la dinámica demográfica de la zona al identificarse, para algunos municipios o localidades seleccionadas de la frontera norte, el efecto que genera la inmigración -a través de la alteración de las estructuras por edad y sexo- en los mercados matrimoniales (Ojeda, 1990) y en los patrones de nupcialidad fronteriza (Quilodrán, 1990).

De esta manera, se han comenzado a estudiar las repercusiones que genera la inmigración sobre algunos de los factores del crecimiento natural 
de la población en la frontera norte de México. El componente del crecimiento natural en la dinámica demográfica de esta zona del país ha representado, a lo largo de las seis décadas entre 1930 y 1990, alrededor del $90.0 \%$ de las tasas anuales de crecimiento total de la población de las seis entidades fronterizas del norte del país (Corona, 1991:149).

Con estos antecedentes, se ha planteado como objetivo general de este trabajo el análisis del impacto indirecto que genera la inmigración hacia el estado de Baja California a través del comportamiento reproductivo de las mujeres migrantes que residen en dicha entidad. Para el logro de ese objetivo, en la primer parte del trabajo se presentan tanto la trayectoria y principales características de la fecundidad en las entidades fronterizas del norte de México, como los rasgos que se han identificado como definitorios del perfil reproductivo de las mujeres migrantes en estudios de carácter nacional y regional. A continuación, el segundo apartado contiene la presentación de los elementos y antecedentes de aplicación del modelo analítico utilizado, las fuentes de información a que se recurre y los resultados obtenidos para el caso de Baja California, los cuales se comentan en un contexto general en la sección de conclusiones.

\section{TENDENCIAS DE LA FECUNDIDAD EN LA FRONTERA NORTE DE MÉXICO}

El comportamiento que se ha observado en las tendencias de la fecundidad de las entidades fronterizas del norte de México (BFNM) a lo largo de los últimos cincuenta años, permite advertir tres rasgos que caracterizan el perfil reproductivo de esta zona del país durante ese periodo.

Un primer rasgo que se puede advertir a partir de la información presentada en el primer panel del cuadro 1 , es el relativo al hecho de que hasta la década de 1950 a 1960, tanto el conjunto como cada una de las EFNM presentaban una tendencia a incrementar sus tasas brutas de natalidad (TBN). ${ }^{1}$ A través de ese proceso de aumento paulatino, la mayoría de las EFNM así como su conjunto pasaron de una situación en la que presentaban TBN menores a las del país y del resto de las entidades (i.e. 1930-1940), a una en la que sus TBN resultaron superiores (i.e. 1950-1960) a las de esos dos últimos agregados.

Esta tendencia creciente de la natalidad en las BFNM, en principio parece haber estado vinculada a un proceso de reducción de la mortalidad que, en su fase más acentuada, se desarrolló una década antes en las EFNM

$1 \mathrm{TBN}=($ Nacimientos del año/Población total a mitad del año $) \times 1000$ 
MOVILIDAD DE POBLACION Y COMPORTAMIENTO REPRODUCTIVO

CUADRO 1. México y estados de la frontera norte. Tasas brutas de natalidad y tasas globales de fecundidad.

\begin{tabular}{|c|c|c|c|c|c|c|}
\hline \multicolumn{7}{|l|}{ Concepto y Área } \\
\hline \multirow[b]{2}{*}{ TBN $(\% o)$} & \multicolumn{6}{|c|}{ Déc a d a } \\
\hline & $1930-40$ & $1940-50$ & $1950-60$ & $1960-70$ & $1970-80$ & $1980-90$ \\
\hline Rep. mexicana & 44.1 & 45.0 & 45.0 & 45.2 & 39.5 & 29.7 \\
\hline Edos. front. nte. & 43.9 & 45.8 & 46.4 & 45.5 & 38.8 & 25.8 \\
\hline Baja California & 41.3 & 44.7 & 48.5 & 46.4 & 34.5 & 24.3 \\
\hline Coahuila & 50.5 & 52.5 & 49.5 & 50.4 & 46.4 & 28.8 \\
\hline Chihuahua & 43.2 & 43.3 & 43.9 & 43.3 & 36.8 & 24.7 \\
\hline Nuevo León & 41.8 & 45.1 & 45.8 & 45.1 & 39.5 & 24.9 \\
\hline Sonora & 42.4 & 48.7 & 50.9 & 49.0 & 39.9 & 25.0 \\
\hline Tamaulipas & 41.1 & 40.4 & 42.8 & 41.4 & 35.7 & 27.2 \\
\hline \multirow[t]{2}{*}{ Otros estados } & 44.1 & 44.9 & 44.7 & 45.1 & 39.6 & 30.5 \\
\hline & \multicolumn{6}{|c|}{ A ñ o } \\
\hline TGF (hijos) & 1952 & 19 & & 1970 & 1980 & 1984 \\
\hline Rep. mexicana & 5.95 & 6. & 49 & 6.51 & 4.91 & 4.41 \\
\hline Baja California & 6.47 & 6. & 63 & 6.07 & 3.83 & 3.61 \\
\hline Coahuila & 6.12 & 6. & 85 & 7.46 & 5.05 & 4.20 \\
\hline Chihuahua & 5.49 & 6. & 11 & 5.65 & 4.18 & 3.41 \\
\hline Nuevo León & 5.27 & 6. & 18 & 5.60 & 4.16 & 3.45 \\
\hline Sonora & 6.21 & 6. & 92 & 6.70 & 4.29 & 3.81 \\
\hline Tamaulipas & 5.37 & 5. & 67 & 5.55 & 4.61 & 2.72 \\
\hline
\end{tabular}

FUENTE: TBM: Corona, 1991:146; TGF: Chávez, 1987:45.

que en el resto del país (Corona, 1991:154). Al reducir anticipadamente su mortalidad, las EFNM también experimentaron de manera temprana los efectos que de ello derivan para la natalidad (Nag, 1980), pues con la disminución de la mortalidad se prolongan los periodos que las parejas se mantienen en unión conyugal, a la vez que decrece el número de las uniones que se disuelven a causa de la viudez. De esta manera, pues, la interacción entre la mortalidad y la natalidad aparece como el factor que probablemente sustentó la tendencia observada en las BFNM hasta los años cincuenta. 
A partir del final de esa década, sin embargo, empieza a manifestarse lo que llegaría a constituir el segundo rasgo que caracteriza al perfil reproductivo de esta área de México. En este caso, la información presentada en el segundo panel del cuadro 1, permite apreciar que las tasas globales de fecundidad (TGF) ${ }^{2}$ correspondientes a las EFNM empiezan a partir de 1960, a descender con la excepción de Coahuila, en tanto que la TGF del país en su conjunto continúa su tendencia ascendente hasta 1970.

Es decir que, al igual que con la mortalidad, en las EFNM se puede observar un inicio del proceso de reducción de la fecundidad anticipado al del conjunto del país. $\mathrm{Al}$ respecto, utilizando indicadores diferentes del aquí comentado y analizando cada una de las entidades federativas de México, Monterrubio et al. (1993) identificaron los 15 estados del país que iniciaron la tercera etapa de su transición demográfica (i.e. el descenso de su fecundidad) con carácter aparentemente definitivo en 1960 o antes. En ese grupo quedaron incluidas cinco de las seis EFNM, reiterándose la excepción antes mencionada para el caso del estado de Coahuila (Monterrubio, et al., 1993: 304-305).

$\mathrm{Al}$ iniciar de manera comparativamente temprana su proceso de reducción de la fecundidad, las EFNM también comenzaron a definir el tercer rasgo que caracteriza su comportamiento reproductivo actual. Este último rasgo, derivado del inicio temprano y de la intensidad con que se desarrollo el proceso de reducción de la fecundidad en esta zona del país, consiste en los bajos niveles que han adquirido los valores de esta variable demográfica en estas entidades de México. Esta particularidad del comportamiento reproductivo en las EFNM ya resulta observable a mediados de la década de los ochenta, tal como lo indican los datos en la última columna de los dos páneles que integran el cuadro 1.

Ahí se puede advertir que, tanto en términos de las TBN como de las TGF, el conjunto y cada una de las seis EFNM presentan niveles de fecundidad menores a los del total nacional y al correspondiente a las entidades federativas no fronterizas. Para 1990 los resultados censales han permitido corroborar esta situación dado que, en función del número promedio de hijos nacidos vivos por mujer, las seis EFNM se ubican entre las 12 entidades con los valores más reducidos al generar promedios entre $2.3 \mathrm{y}$ 2.5 hijos por mujer (INEGI, 1992a:42-43).

En el estudio antes mencionado de Monterrubio et al. (1993) se logró establecer que, de las 15 entidades que iniciaron el descenso de la natalidad

2 TGF = Número de hijos que una mujer tendría si: $i$ ) sobrevive hasta los 50 años, y ii) su fecundidad fuera la misma que la observada en un año para las mujeres de 15 a 49 años de edad. 
por lo menos a partir de la década de los años sesenta, "la mayoría de los estados pertenecientes a este grupo [ $10-$ que incluyen a cinco de las seis $\mathrm{BFNM}$ - ] tienen en la actualidad [i.e. 1990] un grado de desarrollo mayor que la media nacional. Se podría decir que representan al México moderno y más avanzado y en el que la población tiene un mejor nivel de vida" (Monterrubio, et al.: 309).

Los mejores niveles de vida que los autores asocian con el inicio temprano de la reducción de la natalidad y que coinciden con las entidades con los menores niveles de fecundidad (García y Garma, 1979:78), también han sido planteados como parte integrante de los elementos que permiten explicar los movimientos migratorios internos hacia las EFNM (Appendini, et al., 1972:19-20). En el caso específico de la migración de mujeres hacia el norte del país, Espinoza (1984) —utilizando las "historias" migratorias generadas por la Encuesta Mexicana de Fecundidad de 1976- logró mostrar tres elementos que caracterizan a los movimientos hacia las tres regiones (i.e. noroeste, noreste y norte) que incluyen a las seis EFNM.

El primer elemento característico en esas regiones, consiste en que en ellas se observaron las tres proporciones más elevadas de mujeres migrantes (i.e. $80.9 \%, 73.5 \%$ y $72.4 \%$, respectivamente), tanto de las ocho regiones definidas como del total nacional (Espinoza, 1984:330). A la vez, un segundo elemento a mencionar consiste en el hecho de que, entre las tres regiones del norte, se observaron los promedios más altos del número de movimientos migratorios por mujer (e.g. 2.6 y 3.0) para las residentes de localidades de 2,500 a 499,999 habitantes (Espinoza, 1984:333).

Finalmente, los dos rasgos antes mencionados se presentaron acompañados por la característica de que, a diferencia de lo observado para el país en su conjunto, en la mayoría de las comparaciones posibles para las tres regiones del norte de México, las mujeres migrantes en unión presentaron promedios de hijos nacidos vivos superiores a los de las mujeres no-migrantes unidas (Espinoza, 1984:338). Más aún, en el caso específico de la región noroeste -que incluye a Baja California- se logro establecer que, en cinco de los siete grupos quinquenales de edad considerados, las mujeres migrantes presentaban niveles de fecundidad acumulada superiores a los de las mujeres no-migrantes que residían en esta zona del país.

Tomadas en conjunto, las características antes mencionadas han permitido sugerir que a las mujeres migrantes se les considere como un grupo "transicional" (Brambila, 1985:22-57), puesto que hay evidencia de que presentan niveles de fecundidad inferiores a los de sus lugares (rurales) de origen, pero que resultan superiores y contribuyen a elevar los niveles de fecundidad de las áreas (urbanas) de destino. 
Con los antecedentes hasta aquí planteados, y tomando en cuenta que para 1990 el $47.0 \%$ de la población de Baja California no era nativa de la entidad y que entre 1985 y 1990 el estado presentó un saldo neto migratorio positivo de más de 180 mil nuevos residentes (INEGI, 1992a:18) que proceden de entidades en las que aún se observan niveles de fecundidad superiores a los del área de destino (INEGI, 1992a:43) excepto el Distrito Federal, Nuevo León y Quintana Roo; a continuación analizamos el comportamiento reproductivo de las mujeres migrantes y nativas residentes de Baja California.

\section{MIGRACIÓN Y FECUNDIDAD EN BAJA CALIFORNIA}

\section{Antecedentes y elementos del modelo}

El marco conceptual de las "variables intermedias" con el que proponemos estudiar el comportamiento reproductivo de las mujeres migrantes y nativas de Baja California, originalmente fue planteado como un recurso para el análisis sociológico comparativo de la fecundidad por Davis y Blake (1956). Estos autores argumentaron que para poder analizar y explicar no sólo los diferenciales observados en los niveles de fecundidad entre países desarrollados y en desarrollo, sino además para comprender cómo se podían presentar niveles de fecundidad similares entre esos grupos de países a pesar de sus formas claramente diferenciadas de organización social, era necesario recurrir a un conjunto de factores que estando directamente conectados con el proceso de reproducción fuesen, a la vez, aquéllos a través de los cuales - y sólo a través de los cualeslas condiciones culturales pudiesen afectar a la fecundidad (Davis y Blake, 1956:211).

Dada su función mediadora entre las estructuras sociales y los patrones de comportamiento reproductivo, Davis y Blake (1956) denominaron "variables intermedias" al conjunto de factores directamente vinculados al proceso reproductivo. En total, los autores identificaron once variables intermedias las que, a su vez, fueron agrupadas de acuerdo a su relación con lo que denominaron los tres "pasos necesarios" de la reproducción, es decir; el coito, la concepción, y la gestación y el parto.

Las seis variables relacionadas con el primer paso de la reproducción, o factores que afectan la exposición al coito, se subdividieron en aquéllas que gobieman la formación y disolución de las uniones (i.e. edad al inicio de la unión sexual, proporción de mujeres en celibato permanente y porción del periodo reproductivo que se pasa fuera de unión por separación, divorcio o viudez), y aquellos que gobiernan la exposición al coito 
dentro de las uniones (i.e. abstinencia voluntaria, abstinencia involuntaria por enfermedad o separación y frecuencia coital). Para el segundo paso de la reproducción, la concepción, se distinguieron tres factores que afectan la exposición a la concepción (i.e. infertilidad - esterilidad involuntaria, uso o no de métodos anticonceptivos y esterilización voluntaria), en tanto que para la fase de la gestación y el parto se identificaron las dos variables restantes (i.e. mortalidad fetal involuntaria y aborto inducido).

Paralelamente al desarrollo de la propuesta conceptual de Davis y Blake, pero de manera independiente, durante los afios cincuentas L. Henry (1972) desarrollo modelos cuantitativos capaces de simular el comportamiento reproductivo de poblaciones históricas. A partir de los estudios de Henry, se logró identificar un conjunto de factores que afectan a la reproducción y que resultó similar al de las variables intermedias, el cual más recientemente ha sido denominado de los "determinantes próximos" de la fecundidad, para distinguirlos de los determinantes socioeconómicos o "remotos" del comportamiento reproductivo.

La presentación más acabada del modelo de los determinantes próximos, ahora considerada clásica, fue realizada por J. Bongaarts (1978) en un artículo en el que propuso reducir a ocho las variables intermedias - para facilitar su cuantificación- y agruparlas en tres categorías, a saber: factores de exposición, factores del control deliberado de la fecundidad marital, y factores de la fecundidad marital natural (Bongaarts, 1978:106).

En el primer grupo se incluyó únicamente al factor de la proporción de casadas, a través de la cual se cuantifica la proporción de mujeres en edades reproductivas (i.e. de 15 a 49 años) que sostienen relaciones sexuales regularmente, esto es, a todas las mujeres que viven en uniones sexuales. En el grupo de factores del control deliberado de la fecundidad marital se incluyeron la anticoncepción y el aborto inducido para cuantificar, respectivamente, las prácticas deliberadas - dependientes de la paridez- que se ejercen para reducir el riesgo de la concepción (incluyendo la abstinencia y la esterilización), así como las prácticas que deliberadamente interrumpen el curso normal de la gestación.

Por su parte, el grupo de factores relativos a la fecundidad marital natural - denominados de esa forma por considerarse que son los que determinan la fecundidad en poblaciones que no ejercen un control deliberado de la reproducción - se integró por la frecuencia del coito, la esterilidad o infertilidad involuntaria, la mortalidad intrauterina espontánea, la duración del periodo fértil y la infecundabilidad. Este último factor, originalmente no incluido en las variables intermedias, cuantifica la prolongación del periodo de infecundabilidad postparto, atribuible a la duración e intensidad de la lactancia postparto. 
En el artículo antes citado y en trabajos posteriores Bongaarts (1982) logró establecer, con información de poblaciones contemporáneas, que los primeros cuatro factores de la fecundidad marital natural tienen efectos poco significativos - salvo en situaciones excepcionales (Bongaarts:1978;119-120)- tanto en la variación de los niveles de la fecundidad como en la explicación de las diferencias de fecundidad entre poblaciones. Por tal motivo, el modelo de los determinantes próximos de la fecundidad sólo incluye la proporción de mujeres en unión, la proporción de estas últimas que utilizan métodos anticonceptivos, la duración del periodo de lactancia y el recurso del aborto.

Al igual que Davis y Blake, Bongaarts (1978) también propuso que el modelo de los determinantes próximos se utilizara para fines de análisis comparativos de fertilidad entre poblaciones y subgrupos en una población, para así poder establecer a qué variables intermedias se les podían atribuir las diferencias de fecundidad entre las poblaciones que se compararan (Bongaarts, 1978:125).

Sin embargo, a pesar de contar con dicho potencial comparativo, el modelo conceptual no ha sido utilizado con la frecuencia esperada en el rubro de indagación que atiende a la relación entre migración y fecundidad. En el caso de México, por ejemplo, García y Garma (1989) realizó una revisión de la literatura relativa a 75 estudios demográficos del país en los que se utiliza - total o parcialmente-el marco conceptual de las variables intermedias, cuyo resultado permite advertir que lo más cercano a un análisis comparativo es el estudio de los comportamientos reproductivos de las poblaciones urbanas y rurales.

En torno a los diferenciales urbano-rurales, los estudios ahí revisados indican que se observan desigualdades en torno a la edad a la primera unión (i.e. mayor en las zonas urbanas), a la proporción de mujeres fuera de unión conyugal (i.e. mayor en las zonas urbanas), y la duración de la lactancia que es más prolongada en las zonas rurales de México (García y Garma, 1989:221-238). De igual forma el estudio de Welti (1989), en relación específicamente a la práctica anticonceptiva en México, pone en evidencia la ausencia de trabajos que analicen los comportamientos reproductivos de la población en función de su movilidad territorial.

Esta ausencia, en parte explicable debido a la carencia de la información requerida, también resulta difícil de entender a la luz de los resultados que ha arrojado gran parte de la investigación demográfica que ha atendido al tópico mencionado. Al respecto, por ejemplo, Myers y Macisco (1975) al revisar la literatura referida a la relación entre migración y fecundidad mostraron que, aparte del nivel educativo, las variables que más frecuentemente se asociaban con los diferenciales de fecundidad entre migrantes 
y no migrantes correspondian a las estructuras de: edad, estado civil, edad de matrimonio y de las prácticas anticonceptivas.

Ese tipo de resultados, y el hecho de que otros estudios han puesto en evidencia que en el suroeste de los Estados Unidos la fecundidad de las mexicoamericanas es mucho más alta que la de las anglosajonas, le permitieron a Warren (1992) proponer la existencia de un continuum de diferentes niveles de la transición demográfica desde una fecundidad "natural" hasta una "controlada". En ese continuum teórico se asume que México como un todo debe aparecer muy próximo a la situación de fecundidad natural, y alejándose progresivamente de esa situación se ubicarian las seis EFNM, las mujeres mexicoamericanas del suroeste de Estados Unidos que no dominan el inglés, las que sí lo hacen y, finalmente, las anglosajonas que estarian en la situación de fecundidad controlada (Warren, 1992:108).

Para probar su propuesta de que las mexicoamericanas son una población en transición - por aculturación - entre una sociedad con fecundidad natural (i.e. México) y una con fecundidad controlada (i.e. los Estados Unidos), Warren (1992) utiliza información correspondiente a 1979 y recurre al modelo de los determinantes próximos de la fecundidad de Bongaarts (1978). De esa manera, el autor logra sustento para su propuesta y demuestra que, en comparación con México en su conjunto, en 1979 las BFNM presentaban menores niveles en sus TGF, menores proporciones de mujeres en unión, mayores proporciones de estas últimas que utilizan anticonceptivos, edades medianas menores al parto, y menores duraciones del periodo infecundo postparto por lactancia (Warren, 1992:111-116).

Para dar seguimiento a esta forma de aproximación, con la que se advierten evidencias de un comportamiento diferenciado en términos de los determinantes próximos de la fecundidad entre las seis EFNM y las principales áreas de origen de los flujos migratorios que se dirigen hacia esas entidades, a continuación se definen los elementos integrantes del modelo cuantitativo de Bongaarts (i.e. índices de matrimonio, de anticoncepción e infecundabilidad postparto), se describen las fuentes de información a utilizar y se presentan los resultados para el caso de las poblaciones nativas y migrantes de Baja California.

\section{El caso de Baja California}

En el modelo de Bongaarts (1978), la cuantificación del efecto de las variables intermedias o determinantes próximos sobre la fecundidad se realiza a través de la construcción de índices que toman valores de 0 a 1 . El cálculo de los tres ${ }^{3}$ indices a incluir en este análisis se define de la siguiente forma: 
Indice de matrimonio o de las proporciones en union $(\mathrm{Cm})$ :

$\mathrm{Cm}=\mathrm{TGF} / \mathrm{TM}$,

donde:

Tasa global de fecundidad legítima $=\mathbf{\Sigma} f(a) \times 5$;

$\mathrm{f}(\mathrm{a})=$ tasas específicas de fecundidad legítima por grupos quinquena-

les de edad (i.e. hijos nacidos de mujeres en unión $x+4$ / mujeres en unión conyugal $x+4)$.

Tasa de fecundidad marital $(\mathrm{TM})=\Sigma \mathrm{g}(\mathrm{a}) \times 5$;

$g(a)=$ tasas específicas de fecundidad marital por grupos quinquenales de edad (i.e. $\mathrm{f}(\mathrm{a}) \mathrm{x}+4 / \mathrm{m}(\mathrm{a}) \mathrm{x}+4$ );

$\mathrm{m}(\mathrm{a}) \mathrm{x}+4=$ proporción de mujeres en unión del total del grupo de edad.

Indice de anticoncepcion $(\mathrm{Cc})$ :

donde:

$$
\mathrm{Cc}=1-(1.08 \times \mathrm{u} \times \mathrm{e})
$$

1.08 = factor de corrección por esterilidad;

$\mathrm{u}=$ porcentaje de mujeres en unión no embarazadas que usan métodos anticonceptivos o prevalencia;

$\mathrm{e}=\Sigma(\mathrm{i}) / \mathrm{u}$;

$\mathrm{i}=\%$ de usuarias por tipo de método $\times$ estándar de efectividad-uso del método.

Indice de infecundabilidad postparto $(\mathrm{Ci})$ :

donde:

$$
\mathrm{Ci}=20 /(18.5 \times \mathrm{i})
$$

20 = intervalo promedio, en meses, entre nacidos vivos en ausencia de abstinencia postparto y amenorrea por lactancia;

18.5 = intervalo promedio, en meses, entre nacidos vivos en ausencia de lactancia;

$\mathrm{i}=1.753 \times \operatorname{Exp}\left\{(0.1396 \times \mathrm{B})-\left(0.001872 \times \mathrm{B}^{2}\right)\right\}$, donde:

$1.753,0.1396$ y 0.001872 son cocficientes parciales de regresión, y;

B = promedio, en meses, de duración de la lactancia.

A partir de esos tres índices el autor del modelo establece tres medidas de la fecundidad (Bongaarts, 1978; Bongaarts y Potter, 1983), que se definen y relacionan en los siguientes términos:

3 Se excluye el índice de aborto por no disponer de información confiable en relación a este factor, como sucede a nivel nacional (Núflez y Palma, 1990) y en la mayoria de Latinoamérica (Frejka y Atkin, 1990). 
Tasa de fecundidad marital (TM); TM = TGFL $/ \mathrm{Cm}$. Indica el nivel que alcanzarfa la fecundidad, si todas las mujeres en edades reproductivas estuvieran en unión conyugal.

Tasa de fecundidad marital natural ( $\mathrm{TN}) ; \mathrm{TN}=\mathrm{TM} / \mathrm{Cc}$. Indica el nivel que alcanzaria la fecundidad si todas las mujeres en edades reproductivas estuvieran en unión y dejaran de practicar la anticoncepción.

Tasa de fecundidad potencial (TF); $\mathrm{TF}=\mathrm{TN} / \mathrm{Ci}$. Indica $\mathrm{el} \mathrm{nivel} \mathrm{que}$ alcanzaría la fecundidad si todas las mujeres en edades reproductivas estuvieran en unión, no practicaran la anticoncepción y dejaran de practicar la lactancia.

Para la aplicación de este modelo al caso que nos ocupa, hemos recurrido a la información generada por las Encuestas Demográficas de Baja California de 1986 y 1990 (CONEPO-BC, CRIM-UNAM, IS-UABC, 1987. IIS-UABC y CONBPO-BC, 1993). Ambas encuestas se sustentaron en cuatro marcos muestrales independientes (i.e. municipales) de viviendas, los cuales se construyeron ex profeso en 1986 y se actualizaron para la encuesta de 1990. Con ellos se generaron dos muestras probabilísticas polietápicas, seleccionándose como unidades terciarias de nuestro alrededor de 5,000 viviendas para la aplicación de cuestionarios por hogar en cada uno de los afios mencionados.

Estas encuestas, que tienen representatividad a nivel urbano y rural para cada municipio, incluyeron información de vivienda, de inmigrantes temporales, de defunciones en el último affo, de emigración definitiva y características de los residentes habituales. Para este último grupo, entre otros, se captaron datos de edad, sexo, entidad de nacimiento, tiempo de residencia en el municipio, estado civil y —para las mujeres— número de hijos(as) nacidos vivos, fecha de nacimiento del último hijo(a), así como prácticas anticonceptivas y de lactancia. Las muestras de mujeres entre 15 y 49 años de edad fueron de 4,979 y 5,859 en 1986 y 1990, respectivamente, y constituyen la base de información que se analiza a continuación.

A partir de los datos incluidos en los cuadros 3 a 8 se ha integrado el cuadro 2 , en el que se presentan tanto los tres índices del modelo de los determinantes próximos de la fecundidad (i.e. $\mathrm{Cm}, \mathrm{Cc}$ y $\mathrm{Ci}$ ), como los valores estimados para las tasas de fecundidad global (TGF), global legítima (TGFL), marital (TM), marital natural (TN) y la de fecundidad potencial (TF), para el total de México, de Baja California y para las mujeres nativas y migrantes que residen en la última entidad. ${ }^{4}$

\footnotetext{
4 En este trabajo se consideran "nativas" o "ho migrantes" a las mujeres que nacieron en B.C y nunca han carnbiado su lugar de rexidencis. Por tanto, las migrantes incluyen a las mujeres nacidas fuera de la entidad y a las nativas de B.C. que han mignado dentro del estado y/o entre estados.
} 
CUADRO 2. México y B.C. índices de los determinantes próximos y estimaciones de las tasas de fecundidad (1976, 1986, 1990).

\begin{tabular}{lcccccccc}
\hline \multicolumn{2}{l}{$\begin{array}{l}\text { Indices } \\
\text { y tasas }\end{array}$} & \multicolumn{2}{c}{ B.C. total } & \multicolumn{2}{c}{ B.C. nativas } & \multicolumn{2}{c}{ B.C. migrantes } & \multicolumn{2}{c}{ México } \\
& 1990 & 1986 & 1990 & 1986 & 1990 & 1986 & 1986 & 1976 \\
\hline & & & & & & & & \\
Cm & 0.5513 & 0.5942 & 0.4977 & 0.5499 & 0.6019 & 0.6382 & 0.6200 & 0.6100 \\
Cc & 0.3833 & 0.3858 & 0.3291 & 0.3427 & 0.4088 & 0.4027 & 0.4903 & 0.7310 \\
Ci & 0.8988 & 0.9123 & 0.9221 & 0.9339 & 0.8869 & 0.9034 & 0.8105 & 0.8410 \\
TF & 12.48 & 12.77 & 14.37 & 13.81 & 11.69 & 12.66 & 14.01 & 15.28 \\
TN & 11.22 & 11.65 & 13.25 & 12.89 & 10.36 & 11.44 & 11.36 & 12.85 \\
TM & 4.30 & 4.49 & 4.36 & 4.42 & 4.24 & 4.61 & 5.57 & 9.39 \\
TGFL & 2.37 & 2.67 & 2.17 & 2.43 & 2.55 & 2.94 & 3.45 & 5.73 \\
TGF & 2.59 & 2.97 & 2.37 & 2.75 & 2.80 & 3.26 & 3.84 & 6.31 \\
& & & & & & & & \\
\hline
\end{tabular}

FUENTE: México: SPP, 1979; SSA, 1989. Baja California: cuadros 3 a 8.

El primer elemento a seffalar en relación con los datos incluidos en el cuadro 2, consiste en que a través de las TGF se advierte que, tanto en Baja California como en el país en su conjunto, la fecundidad sostuvo sus tendencias de reducción durante los ochentas. En el país la TGF se redujo de 4.91 a 3.89 entre 1980 y 1986, en tanto que en ese mismo lapso la TGF de la entidad pasó de 3.83 a 2.97 (cuadros 1 y 2). De esta forma, a la vez, se incrementó el diferencial entre los niveles de fecundidad del país y de Baja California, dado que en 1980 la TGF de la entidad representaba $78.0 \%$ de la nacional en tanto que para 1986 la proporción se redujo al $76.4 \%$.

El diferencial de fecundidad que se puede observar entre México y Baja California en 1986, se reproduce entre la población de nativas y migrantes de la entidad, puesto que la TGF de las primeras (i.e. 2.75) resulta menor que la de las migrantes (i.e. 3.26). En este caso, sin embargo, también se observa que las diferencias en el nivel de fecundidad entre las migrantes y nativas de la entidad permanecen virtualmente constantes entre 1986 y 1990, ya que aún cuando para el último af́o mencionado las TGF de ambas poblaciones se han reducido (i.e. a 2.80 y 2.37 respectivamente), la fecundidad de las nativas representa en ambas fechas alrededor del $84.5 \%$ de la de las migrantes.

Un primer factor involucrado en las diferencias entre la fecundidad de las mujeres migrantes y la de las nativas de la entidad, consiste en los distintos patrones de fecundidad ilegítima (i.e. nacimientos fuera de unión) 
que se observan para estos grupos. Al respecto, la comparación de los dos últimos renglones del cuadro 2 permite advertir que la fecundidad ilegítima (i.e. TGF-TGFL) de las migrantes represento $9.8 \%$ y $8.9 \%$ de su TGF en 1986 y 1990, respectivamente, en tanto que la de las mujeres nativas alcanzaba el $11.6 \%$ y $8.4 \%$ de sus correspondientes TGF en las dos fechas mencionadas. De esta manera, a la vez que observa una reducción de la fecundidad ilegítima en ambos grupos, se hace evidente que mientras la de las migrantes se reduce en menos de $10.0 \%$ entre 1986 y 1990, la del grupo de mujeres nativas lo hace en más de $27.5 \%$.

Otro factor que sustenta las diferencias observadas en los niveles de fecundidad entre las mujeres migrantes y nativas de Baja California es el relativo a los patrones diferenciales de nupcialidad que manifiestan cada uno de esos grupos. En este sentido, por una parte, los datos de mujeres unidas en los cuadros 3 y 4 así como su representación en la figura 1 , muestran que en cinco de los siete grupos quinquenales de edad considerados, las migrantes presentan mayores proporciones de mujeres en unión que el grupo de nativas, tanto en 1986 como en 1990.

Además, por otra parte, la misma figura permite advertir que, en lo general, las diferencias en las proporciones de mujeres en unión por grupos quinquenales se han acentuado entre 1986 y 1990, dado que las líneas relativas a migrantes y nativas para 1990 se encuentran más separadas que las correspondientes a 1986. En términos de edades al matrimonio, las diferencias para 1990 implican que la edad media al matrimonio ${ }^{5}$ de las mujeres migrantes (i.e. 21.7) es casi dos afios inferior que la de las mujeres nativas (i.e. 23.6).

La influencia que ejercen los patrones de nupcialidad observados sobre la fecundidad tanto de migrantes como de nativas, se expresa en los diferentes valores que adquieren los índices de matrimonio $(\mathrm{Cm})$ que se han incluido en el primer renglón del cuadro 2. Ahí se puede advertir que, mientras el grupo de migrantes conserva alrededor del $60.0 \%$ de su fecundidad marital (TM) una vez descontado su patrón de nupcialidad tanto en 1986 como en 1990, las mujeres nativas reducen su TM a un nivel de $55.0 \%$ en 1986 y de menos de $50.0 \%$ en 1990.

Así, los datos anteriores confirman no sólo que los patrones de nupcialidad han sido y se mantienen diferentes, sino que las diferencias y sus efectos sobre la fecundidad se han acrecentado entre 1986 y 1990, puesto que en ese lapso los valores de $\mathrm{Cm}$ se redujeron en $5.7 \%$ para las mujeres migrantes, en tanto que el grupo de nativas redujo su $\mathrm{Cm}$ en $9.5 \%$.

5 Edad media al matrimonio $=15+\left\{\left(\left(\mathrm{n} \times \Sigma_{\mathrm{n}} \mathrm{S}_{\mathrm{i}}\right)-\left(35 \times \mathrm{S}_{50}\right)\right) / 1-\mathrm{S}_{50}\right\}$ donde: $\mathrm{nSi}=$ proporciones de solteras por grupo de edad quinquenal, $\mathrm{S}_{50}=\left(\mathrm{S}_{45-49}+\mathrm{S} 50-54\right) / 2$. 
CUADRO 3. B.C. población total, nativa y migrante: determinantes próximos de la fecundidad, índices de matrimonio(1986).

\begin{tabular}{lcccccc}
\hline $\begin{array}{c}\text { Grupo } \\
\text { de } \\
\text { edad }\end{array}$ & $\begin{array}{c}\text { (1) } \\
\text { Total } \\
\text { mujeres }\end{array}$ & $\begin{array}{c}\text { (2) } \\
\text { En } \\
\text { unión }\end{array}$ & $\begin{array}{c}\text { (3) } \\
\text { Nacimientos } \\
\text { en unión }\end{array}$ & $\begin{array}{c}\text { \%Unidas } \\
\text { m(a) } \\
(2 / 1)\end{array}$ & $\begin{array}{c}\text { TEFL } \\
\text { f(a) } \\
(3 / 1)\end{array}$ & $\begin{array}{c}\text { g(a) } \\
\text { (fa/ma) }\end{array}$ \\
\hline $\begin{array}{l}\text { A. Poblaci6n total. } \\
\text { Cm = fnd. de matrimonio = TGFL/TM=0.5942 }\end{array}$ & & & & \\
$15-19$ & 1164 & 152 & 52 & 0.1306 & 0.0447 & 0.1820 \\
$20-24$ & 1040 & 507 & 123 & 0.4875 & 0.1183 & 0.2426 \\
$25-29$ & 785 & 584 & 119 & 0.7439 & 0.1516 & 0.2038 \\
$30-34$ & 668 & 549 & 78 & 0.8219 & 0.1168 & 0.1421 \\
$35-39$ & 539 & 442 & 32 & 0.8200 & 0.0594 & 0.0724 \\
$40-44$ & 423 & 337 & 10 & 0.7967 & 0.0236 & 0.0297 \\
$45-49$ & 360 & 272 & 7 & 0.7556 & 0.0194 & 0.0257 \\
& 4979 & 2843 & 421 & & TGFL & TM \\
& & & & 2.669 & 4.491
\end{tabular}

B. Poblacion nativa

$\mathrm{Cm}=$ Ind. de matrimonio $=\mathrm{TGFL} / \mathrm{TM}=0.5499$

$\begin{array}{rrrrrrr}15-19 & 684 & 76 & 23 & 0.1111 & 0.0336 & 0.1797 \\ 20-24 & 483 & 217 & 52 & 0.4493 & 0.1077 & 0.2396 \\ 25-29 & 305 & 209 & 38 & 0.6852 & 0.1246 & 0.1818 \\ 30-34 & 195 & 143 & 13 & 0.7333 & 0.0667 & 0.0909 \\ 35-39 & 113 & 90 & 8 & 0.7965 & 0.0708 & 0.0889 \\ 40-44 & 50 & 41 & 2 & 0.8200 & 0.0400 & 0.0488 \\ 45-49 & 46 & 36 & 2 & 0.7826 & 0.0435 & 0.0556 \\ & 1876 & 812 & 138 & & \text { TGFL } & \text { TM } \\ & 18 & & & & 2.434 & 4.427\end{array}$

C. Población migrante

$\mathrm{Cm}=$ fnd. de matrimonio $=\mathrm{TGFL} / \mathrm{TM}=0.6382$

\begin{tabular}{rrrrrrr}
$15-19$ & 480 & 76 & 29 & 0.1583 & 0.0604 & 0.1836 \\
$29-24$ & 557 & 290 & 71 & 0.5206 & 0.1275 & 0.2448 \\
$25-29$ & 480 & 375 & 81 & 0.7813 & 0.1688 & 0.2160 \\
$30-34$ & 473 & 406 & 65 & 0.8584 & 0.1374 & 0.1601 \\
$35-39$ & 426 & 352 & 24 & 0.8263 & 0.0563 & 0.0682 \\
$40-44$ & 373 & 296 & 8 & 0.7936 & 0.0214 & 0.0270 \\
$45-49$ & 314 & 236 & 5 & 0.7516 & 0.0159 & 0.0212 \\
& 3103 & 2031 & 283 & & TGFL & TM \\
& & & & & 2.939 & 4.605 \\
\hline
\end{tabular}

FUENTE: CONEPO-BC, CRIM-UNAM, IIS-UABC,1987; IIS-UABC Y CONEPO-BC,1993. NOTA: $g(a)$ para el grupo de 15-19 se calculó como: $(0.75 \times$ g20-24). 


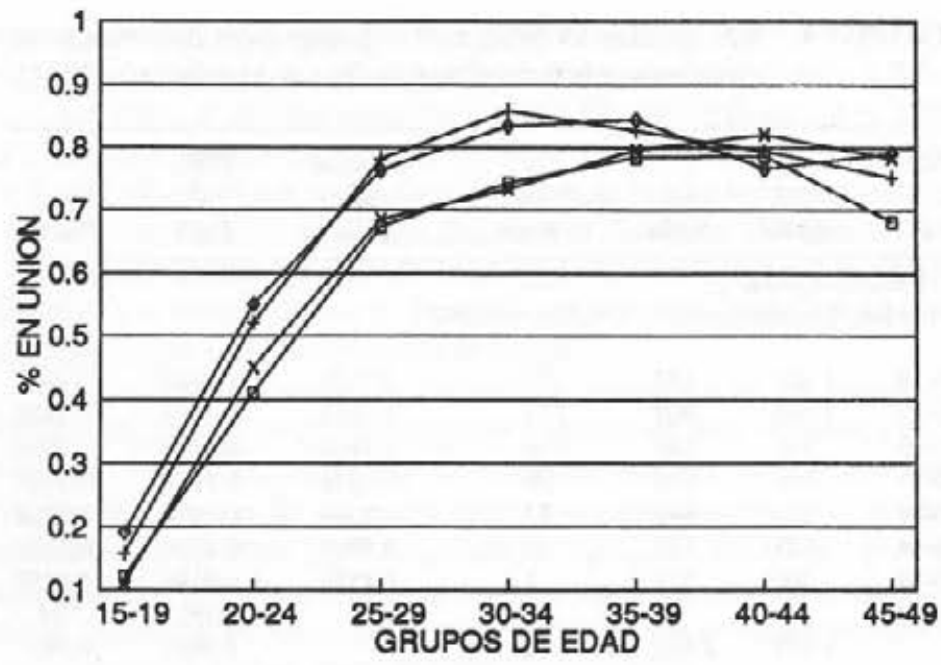

๑- Nativas $1990 \rightarrow$ Migrantes $1990 \rightarrow$ Nativas 1986 —- Migrantes 1986

Figura 1. B.C. proporción de mujeres en unión por grupos de edad. E.D.B.C. 1986 y 1990.

Fuente: Cuadros 3 y 4.

El tercer factor en que se sustentan las diferencias en el comportamiento reproductivo de migrantes y nativas en la entidad, es el correspondiente a los patrones de utilización de métodos anticonceptivos que se observan entre estas dos poblaciones, tanto en lo referido a la intensidad con que se recurre a los métodos como a la particular combinación de métodos que define el perfil de cada uno de los grupos.

En relación al primero de esos dos rubros, la información presentada en las figuras 2 y 3 permite observar que, mientras en 1986 sólo en dos grupos de edad (i.e. de 40 a 44 y 45 a 49 ) las proporciones de mujeres migrantes en unión que utilizaban métodos anticonceptivos superaban a las correspondientes a los grupos de nativas, para 1990 los porcentajes de mujeres nativas en unión que son usuarias de métodos anticonceptivos son superiores a los de las migrantes en los siete grupos de edad considerados. Se debe notar, además, que la intensificación de las diferencias en los patrones de prevalencia en el uso de anticonceptivos entre nativas y migrantes de 1986 a 1990, se origina en las reducciones más acentuadas que se observan en las proporciones de mujeres migrantes en unión que utilizan anticonceptivos, particularmente en los primeros cuatro grupos de edad. 
CUADRO 4. B.C. población total, nativa y migrante: determinantes próximos de la fecundidad, índices de matrimonio (1990).

\begin{tabular}{lcccccccc}
\hline $\begin{array}{l}\text { Grupo } \\
\text { de }\end{array}$ & $\begin{array}{c}\text { Total } \\
\text { edad }\end{array}$ & $\begin{array}{c}\text { en } \\
\text { mujeres }\end{array}$ & $\begin{array}{c}\text { nac. } \\
\text { unión en unión total }\end{array}$ & $\begin{array}{c}\text { m(a) } \\
(2 / 1)\end{array}$ & $\begin{array}{c}\text { (4) } \\
\text { manidas }\end{array}$ & $\begin{array}{c}\text { TEFL } \\
(3 / 1)\end{array}$ & $\begin{array}{c}\text { g(a) } \\
(\mathrm{fa} / \mathrm{ma})\end{array}$ \\
\hline
\end{tabular}

A. Población total.

$\mathrm{Cm}=$ Ind. de matrimonio $=\mathrm{TGFL} / \mathrm{TM}=0.5513$

$\begin{array}{rrrrrrrrr}15-19 & 1379 & 203 & 70 & 85 & 0.1472 & 0.0616 & 0.0508 & 0.2042 \\ 20-24 & 1180 & 562 & 153 & 171 & 0.4763 & 0.1449 & 0.1297 & 0.2722 \\ 25-29 & 927 & 671 & 127 & 135 & 0.7238 & 0.1456 & 0.1370 & 0.1893 \\ 30-34 & 776 & 621 & 79 & 84 & 0.8003 & 0.1082 & 0.1018 & 0.1272 \\ 35-39 & 642 & 530 & 29 & 31 & 0.8255 & 0.0483 & 0.0452 & 0.0547 \\ 40-44 & 505 & 389 & 4 & 4 & 0.7703 & 0.0079 & 0.0079 & 0.0103 \\ 45-49 & 450 & 348 & 1 & 1 & 0.7733 & 0.0022 & 0.0022 & 0.0029 \\ & & & & & & \text { TGF } & \text { TGFL } & \text { TM } \\ & 5859 & 3324 & 463 & 511 & & 2.594 & 2.373 & 4.304\end{array}$

B. Población nativa

$\mathrm{Cm}=$ Ind. de matrimonio $=\mathrm{TGFL} / \mathrm{TM}=0.4977$

$\begin{array}{rrrrrrrrr}15-19 & 868 & 105 & 39 & 45 & 0.1210 & 0.0518 & 0.0449 & 0.2083 \\ 20-24 & 616 & 252 & 70 & 81 & 0.4091 & 0.1315 & 0.1136 & 0.2778 \\ 25-29 & 408 & 275 & 54 & 57 & 0.6740 & 0.1397 & 0.1324 & 0.1964 \\ 30-34 & 284 & 211 & 29 & 31 & 0.7430 & 0.1092 & 0.1021 & 0.1374 \\ 35-39 & 194 & 152 & 8 & 8 & 0.7835 & 0.0412 & 0.0412 & 0.0526 \\ 40-44 & 112 & 88 & 0 & 0 & 0.7857 & 0.0000 & 0.0000 & 0.0000 \\ 45-49 & 69 & 47 & 0 & 0 & 0.6812 & 0.0000 & 0.0000 & 0.0000 \\ & & & & & & \text { TGF } & \text { TGFL } & \text { TM } \\ & 2551 & 1130 & 200 & 222 & & 2.367 & 2.171 & 4.363\end{array}$

C. Población migrante

$\mathrm{Cm}=$ Ind. de matrimonio $=\mathrm{TGFL} / \mathrm{TM}=0.6019$

$\begin{array}{rrrrrrrrr}15-19 & 511 & 98 & 31 & 40 & 0.1918 & 0.0783 & 0.0607 & 0.2008 \\ 20-24 & 564 & 310 & 83 & 90 & 0.5496 & 0.1596 & 0.1472 & 0.2677 \\ 25-29 & 519 & 396 & 73 & 78 & 0.7630 & 0.1503 & 0.1407 & 0.1843 \\ 30-34 & 492 & 410 & 50 & 53 & 0.8333 & 0.1077 & 0.1016 & 0.1220 \\ 35-39 & 448 & 378 & 21 & 23 & 0.8438 & 0.0513 & 0.0469 & 0.0556 \\ 40-44 & 393 & 301 & 4 & 4 & 0.7659 & 0.0102 & 0.0102 & 0.0133 \\ 45-49 & 381 & 301 & 1 & 1 & 0.7900 & 0.0026 & 0.0026 & 0.0033 \\ & & & & & & \text { TGF } & \text { TGFL } & \text { TM } \\ & 3308 & 2194 & 263 & 289 & & 2.800 & 2.549 & 4.235\end{array}$

FUENTE: CONEPO-BC, CRIM-UNAM, IIS-UABC, 1987; IIS-UABC Y CONEPO-BC,1993. NOTA: $g(a)$ para el grupo de 15-19 se calculó como: $(0.75 \times$ g20-24). 


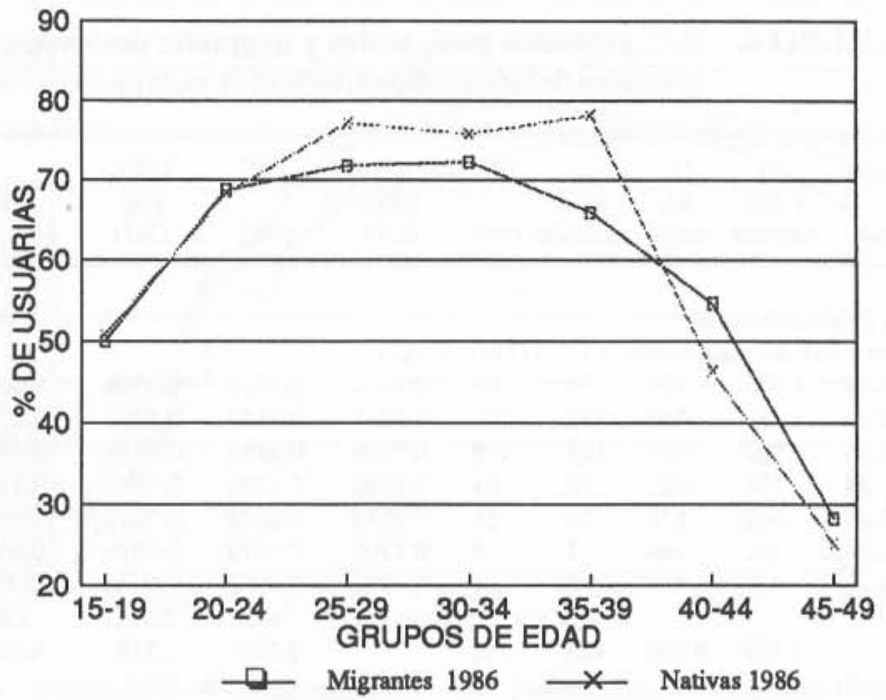

FIGURA 2. B. C. porcentaje de mujeres en unión usuarias de planificación familiar. E.D.B.C. 1986.

Fuente: Cuadros 6 y 7.

Aunado a este comportamiento diferente en la intensidad de la utilización de anticonceptivos -que se manifiesta con proporciones totales crecientes de mujeres nativas en unión que utilizan anticonceptivos de 1986 a 1990 (i.e. $68.2 \%$ y $68.8 \%$ ), y proporciones totales decrecientes para las migrantes (i.e. $61.7 \%$ y $60.1 \%$ ) en ese mismo lapso (ver cuadros 6 y 7) - la información del segundo panel de los cuadros 6 y 7, proporciona evidencia de marcadas discrepancias en el peso que adquieren los diferentes métodos en las prácticas anticonceptivas de los grupos de nativas y migrantes, tanto en 1986 como en 1990.

En este caso, los datos mencionados indican la presencia de dos rasgos característicos. El primero corresponde al hecho de que, tanto en 1986 como en 1990, las mujeres migrantes presentan un patrón de utilización de anticonceptivos altamente concentrado en métodos definitivos (e.g. $44.4 \%$ entre esterilización femenina y vasectomía en 1990), el cual contrasta con una mayor diversificación hacia métodos temporales de control de la fecundidad por parte del grupo de las nativas (e.g. en $199066.4 \%$ utilizan métodos no definitivos).

El segundo rasgo que se advierte en esos mismos datos consiste, por una parte, en el marcado incremento que se observa de 1986 a 1990 entre nativas y migrantes en la utilización de métodos anticonceptivos definitivos 


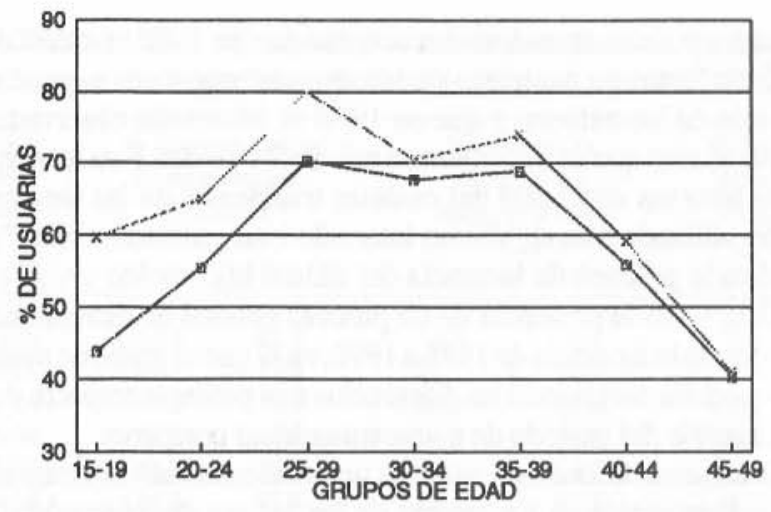

$\square$ Migrantes $1990 \longrightarrow$ Nativas 1990

FIGURA 3. B.C. porcentaje de mujeres en unión usuarias de planificación familiar. E.D.B.C. 1990. Fuente: Cuadros 6 y 7.

en detrimento de métodos como los ciclos orales y los dispositivos intrauterinos (i.e. DIU) y, por otra parte, a la vez aumenta la utilización de condones que contribuye de manera significativa a la elevación de la presencia de los métodos que involucran activamente a los hombres.

La combinación de los patrones de intensidad y de combinación de tipos de métodos que se observan para los grupos de nativas y migrantes, se expresa sumariamente en los índices de anticoncepción (Cc) que se presentan en el segundo renglón del cuadro 2 . Los dos pares de índices referidos a nativas y migrantes - correspondientes a 1986 y 1990 reiteran el proceso de ampliación de las diferencias en las prácticas anticonceptivas de los dos grupos, a la vez que muestran que, mientras que las mujeres migrantes reducen su fecundidad natural (TN) a alrededor del $40.0 \%$ por efecto de sus perfiles de anticoncepción en las dos fechas mencionadas (e.g. de 10.36 a 4.24 en 1990), el grupo de mujeres nativas réduce su TN a menos de $33.0 \%$ en 1990 (i.e. de 13.25 a 4.36) debido a sus prácticas anticonceptivas.

El cuarto y último factor en el que el modelo utilizado permite identificar diferencias en los comportamientos reproductivos de los grupos de migrantes y nativas de Baja California, es el correspondiente a los patrones de lactancia postparto que caracterizan a cada una de esas poblaciones. Al respecto, la información presentada en la primer columna del cuadro 8 indica que, tanto en 1986 como en 1990 , las prácticas de amamantamiento de nativas y migrantes difieren sustantivamente. 
En efecto, los datos mencionados seffalan que en 1986 la media de la duración de la lactancia postparto de las mujeres migrantes superaba en 1.8 meses a la de las nativas, y que en 1990 el diferencial observado no sólo se sostiene sino que se incrementa a más de 2.0 meses. Esta tendencia, aún con las reservas derivadas del carácter transversal de las fuentes de información utilizada para el "último intervalo intergenésico abierto" que corresponde a la práctica de lactancia del último hijo nacido vivo, ${ }^{6}$ es al menos indicativa de la presencia de un proceso general de incremento de las duraciones de la lactancia de 1986 a 1990, en el que el grupo de mujeres migrantes continúa ampliando las diferencias que presenta respecto de las nativas en materia del periodo de amamantamiento postparto.

Las diferencias en la media de meses de lactancia entre nativas y migrantes, dan pie a la divergencia en los valores de los índices de infecundabilidad postparto (Ci) que se observan en el tercer renglón del cuadro 2. Los valores de dichos índices, para 1986 y 1990, muestran que mientras la fecundidad potencial (TF) de las mujeres migrantes ya se reduce a menos del $90.0 \%$ (i.e. de 11.69 a 10.36) debido a los efectos de la lactancia en 1990, la TF de las mujeres nativas no se reduce por debajo del $90.0 \%$ en ninguna de las dos fechas mencionadas dados sus cortos periodos de lactancia.

En conjunto, los determinates próximos de la fecundidad antes descritos generan dos perfiles de comportamiento reproductivo notoriamente diferenciados para los grupos de migrantes y nativas de Baja California. $\mathrm{Al}$ respecto, la información del cuarto al séptimo renglón del cuadro 2 y su representación en la figura 4, permiten advertir las magnitudes absolutas de las diferencias en los cuatro indicadores de la fecundidad considerados (i.e. TF, TN, TM y TFGL).

Para analizar estas diferencias entre grupos, sin embargo, se ha establecido (Singh, et al., 1985) que resulta más pertinente realizar la descomposición de la razón de las TGFL (i.e. TGFLMig / TGFLNat), dado que esto permite el cálculo ${ }^{7}$ del porcentaje que las diferencias en cada uno de los índices y la TF aportan a la diferencia entre las TGFL de los grupos comparados.

\footnotetext{
6 Este tipo de información puede presentar "efectos de selectividad", debido a que la probabilidad de que un "intervalo abierto" sea detectado en una encuesta aplicada una sola vez es proporcional a la duración del intervalo, y por ello las mujeres con intervalos más prolongados tienen may or probabilidad de ser incluidas y su práctica de lactancia corresponde a un periodo anterior al de la media (ver Page et al., 1982:15-22).

La contribución proporcional de los índices y la TP se calcula con la siguiente fórmula:

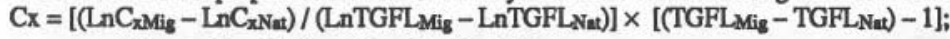

Donde: $\mathrm{Cx}=$ Indices $\mathrm{Cm}, \mathrm{Cc}$, Ci y TF;

Mig $=$ migrantes

Nat $=$ nativas
} 
CUADRO 5. B.C. población total: determinantes próximos de la fecundidad, índices de anticoncepción (1986 y 1990).

\begin{tabular}{cccccccc}
\hline $\begin{array}{c}\text { Grupo } \\
\text { de } \\
\text { edad }\end{array}$ & $\begin{array}{c}\text { \% Mujeres unidas } \\
\text { no embarazadas } \\
\text { usuarias }\end{array}$ & \multicolumn{2}{c}{$\begin{array}{c}\text { Estructura de } \\
\text { edad de las } \\
\text { mujeres unidas } \\
(1)\end{array}$} & \multicolumn{2}{c}{$\begin{array}{c}\text { (2) } \\
\text { (1) }\end{array}$} & \multicolumn{3}{c}{$\begin{array}{c}\text { \% Ponderado de } \\
\text { mujeres unidas } \\
\text { N.E. usuarias } \\
((1 \times 2) / 100)\end{array}$} \\
\hline $15-19$ & 5086 & 1990 & 1986 & 1990 & 1986 & 1990 \\
\hline $20-24$ & 68.5 & 52.5 & 4.9 & 5.4 & 2.5 & 2.8 \\
$25-29$ & 73.8 & 74.1 & 16.7 & 15.9 & 11.4 & 9.5 \\
$30-34$ & 73.2 & 68.6 & 19.5 & 19.2 & 14.9 & 14.2 \\
$35-39$ & 68.4 & 70.3 & 16.0 & 16.8 & 14.3 & 13.0 \\
$40-44$ & 53.6 & 56.6 & 12.5 & 12.5 & 6.7 & 7.1 \\
$45-49$ & 27.8 & 40.4 & 10.2 & 11.2 & 2.8 & 4.5 \\
Promedio & & & & & & & \\
Reportado & 59.4 & 60.3 & \% de usuarias $=$ & 63.57 & 62.98 \\
\hline
\end{tabular}

$1986 \mathrm{Cc}=$ Ind. de anticoncepción $=1-(1.08 \times \mathrm{e} \times \mathrm{u})=0.3858$

$(\mathrm{u})=\%$ prevalencia $=63.57 ;(\mathrm{e})=0.8946$

$1990 \mathrm{Cc}=$ Ind. de anticoncepción $=1-(1.08 \times \mathrm{e} \times \mathrm{u})=0.3833$

$(\mathrm{u})=\%$ prevalencia $=62.98 ;(\mathrm{e})=0.9066$

\begin{tabular}{|c|c|c|c|c|c|}
\hline \multirow[t]{2}{*}{ Método } & \multicolumn{2}{|c|}{$\begin{array}{l}\text { \% de usuarios } \\
\text { por método } \\
\text { (1) }\end{array}$} & \multirow{2}{*}{$\begin{array}{c}\text { Estandard de } \\
\text { efectividad } \\
\text { usuario } \\
\text { (2) }\end{array}$} & \multicolumn{2}{|c|}{$\begin{array}{c}\mathrm{e}(\mathrm{i}) \\
(\mathrm{u} \times(1) \times(2))\end{array}$} \\
\hline & 1986 & 1990 & & 1986 & 1990 \\
\hline Esteriliza & 31.5 & 38.8 & 1.00 & 0.20025 & 0.24437 \\
\hline Vasectomia & 1.1 & 1.5 & 1.00 & 0.00699 & 0.00945 \\
\hline Pastillas & 40.1 & 34.0 & 0.90 & 0.22943 & 0.19273 \\
\hline Inyecciones & 7.9 & 7.8 & 0.70 & 0.03515 & 0.03439 \\
\hline D.I.U. & 11.4 & 9.6 & 0.95 & 0.06885 & 0.05744 \\
\hline Espumas & 0.7 & 0.5 & 0.70 & 0.00311 & 0.00220 \\
\hline Condón & 2.1 & 3.7 & 0.70 & 0.00934 & 0.01631 \\
\hline Diafragma & 0.3 & 0.3 & 0.70 & 0.00133 & 0.00132 \\
\hline Ritmo & 2.4 & 0.0 & 0.70 & 0.01068 & 0.00000 \\
\hline Retirq & 0.5 & 0.0 & 0.70 & 0.00222 & 0.00000 \\
\hline Otros & 0.3 & 2.9 & 0.70 & 0.00133 & 0.01279 \\
\hline N.E. & 1.6 & 0.8 & 0.00 & 0.00000 & 0.00000 \\
\hline Total & 100 & 100 & $(\mathrm{e})=\operatorname{SUMe}(\mathrm{i})$ & $=0.8946$ & 0.9066 \\
\hline
\end{tabular}

FUENTE: CONEPO-BC, CRIM-UNAM, IIS-UABC, 1987; IIS-UABC Y CONEPO-BC,1993.

*: En 1990 "otros" incluye ritmo y retiro. 
CUADRO 6. B.C. población nativa: determinantes próximos de la fecundidad, índices de anticoncepción (1986 y 1990).

\begin{tabular}{|c|c|c|c|c|c|c|}
\hline \multirow[t]{2}{*}{$\begin{array}{l}\text { Grupo } \\
\text { de } \\
\text { edad }\end{array}$} & \multicolumn{2}{|c|}{$\begin{array}{l}\% \text { Mujeres unidas } \\
\text { no embarazadas } \\
\text { usuarias } \\
\text { (1) }\end{array}$} & \multicolumn{2}{|c|}{$\begin{array}{l}\text { Estructura de } \\
\text { edad de las } \\
\text { mujeres unidas } \\
\text { (2) }\end{array}$} & \multicolumn{2}{|c|}{$\begin{array}{c}\text { \% Ponderado de } \\
\text { mujeres unidas } \\
\text { N.E. usuarias } \\
((1 \times 2) / 100)\end{array}$} \\
\hline & 1986 & 1990 & 1986 & 1990 & 1986 & 1990 \\
\hline $15-19$ & 50.7 & 59.6 & 8.9 & 8.9 & 4.5 & 5.3 \\
\hline $20-24$ & 68.2 & 64.8 & 26.3 & 21.3 & 17.9 & 13.8 \\
\hline $25-29$ & 77.2 & 79.8 & 25.7 & 23.7 & 19.8 & 18.9 \\
\hline $30-34$ & 75.8 & 70.5 & 17.6 & 19.0 & 13.3 & 13.4 \\
\hline $35-39$ & 78.2 & 73.8 & 11.5 & 14.5 & 9.0 & 10.7 \\
\hline $40-44$ & 46.2 & 59.0 & 5.2 & 8.3 & 2.4 & 4.9 \\
\hline $45-49$ & 25.0 & 40.9 & 4.8 & 4.4 & 1.2 & 1.8 \\
\hline Promedio & & & & & & \\
\hline Reportado & 60.2 & 64.1 & \multicolumn{2}{|c|}{$\%$ de usuarias $=$} & 68.23 & 68.81 \\
\hline
\end{tabular}

$1986 \mathrm{Cc}=$ fnd. de anticoncepción $=1-(1.08 \times \mathrm{e} \times \mathrm{u})=0.3427$

(u) $=\%$ prevalencia $=68.23 ;(e)=0.8921$

$1990 \mathrm{Cc}=$ Índ. de anticoncepción $=1-(1.08 \times \mathrm{e} \times \mathrm{u})=0.3291$

$(u)=\%$ prevalencia $=68.81 ;(e)=0.9027$

\begin{tabular}{|c|c|c|c|c|c|}
\hline \multirow{2}{*}{ Método } & \multicolumn{2}{|c|}{$\begin{array}{l}\% \text { de usuarios } \\
\text { por método } \\
\text { (1) }\end{array}$} & \multirow{2}{*}{$\begin{array}{l}\text { Estandard de } \\
\text { efectividad } \\
\text { usuario } \\
\text { (2) }\end{array}$} & \multicolumn{2}{|c|}{$\begin{array}{c}\mathrm{e}(\mathrm{i}) \\
(\omega \times(1) \times(2))\end{array}$} \\
\hline & 1986 & 1990 & & 1986 & 1990 \\
\hline Esteriliza. & 23.6 & 32.0 & 1.00 & 0.16101 & 0.22020 \\
\hline Vasectomía & 0.8 & 1.3 & 1.00 & 0.00546 & 0.00895 \\
\hline Pastillas & 46.6 & 40.8 & 0.90 & 0.28614 & 0.25268 \\
\hline Inyecciones & 9.6 & 9.0 & 0.70 & 0.04585 & 0.04335 \\
\hline D.I.U. & 14.2 & 9.6 & 0.95 & 0.09204 & 0.06276 \\
\hline Espumas & 0.4 & 0.3 & 0.70 & 0.00191 & 0.00145 \\
\hline Condón & 1.2 & 4.1 & 0.70 & 0.00573 & 0.01975 \\
\hline Diafragma & 0.2 & 0.3 & 0.70 & 0.00096 & 0.00145 \\
\hline Ritmo & 1.6 & 0.0 & 0.70 & 0.00764 & 0.00000 \\
\hline Retire & 0.2 & 0.0 & 0.70 & 0.00096 & 0.00000 \\
\hline Otros" & 0.2 & 2.2 & 0.70 & 0.00096 & 0.01060 \\
\hline N.E. & 1.6 & 0.3 & 0.00 & 0.00000 & 0.00000 \\
\hline Total & 100 & 100 & $(e)=S U M e(i)$ & $=0.8921$ & 0.9027 \\
\hline
\end{tabular}

FUENTE: CONEPO-BC, CRIM-UNAM, IIS-UABC, 1987; IIS-UABC y CONEPO-BC,1993.

*: En 1990 "otros" incluye ritmo y retiro. 
CUADRO 7. Baja California población migrante: determinantes próximos de la fecundidad, índices de anticoncepción (1986 y 1990).

\begin{tabular}{|c|c|c|c|c|c|c|}
\hline \multirow[t]{2}{*}{$\begin{array}{l}\text { Grupo } \\
\text { de } \\
\text { edad }\end{array}$} & \multicolumn{2}{|c|}{$\begin{array}{l}\% \text { Mujeres unidas } \\
\text { no embarazadas } \\
\text { usuarias } \\
\text { (1) }\end{array}$} & \multicolumn{2}{|c|}{$\begin{array}{l}\text { Estructura de } \\
\text { edad de las } \\
\text { mujeres unidas } \\
\text { (2) }\end{array}$} & \multicolumn{2}{|c|}{$\begin{array}{c}\% \text { Ponderado de } \\
\text { mujeres unidas } \\
\text { N.E. usuarias } \\
((1 \times 2) / 100)\end{array}$} \\
\hline & 1986 & 1990 & 1986 & 1990 & 1986 & 1990 \\
\hline $15-19$ & 50.0 & 43.8 & 3.3 & 3.7 & 1.6 & 1.6 \\
\hline $20-24$ & 68.7 & 55.4 & 12.9 & 13.1 & 8.9 & 7.3 \\
\hline $25-29$ & 71.8 & 70.2 & 18.1 & 17.0 & 13.0 & 11.9 \\
\hline $30-34$ & 72.3 & 67.6 & 20.2 & 19.0 & 14.6 & 12.8 \\
\hline $35-39$ & 65.9 & 68.8 & 17.7 & 18.0 & 11.7 & 12.4 \\
\hline $40-44$ & 54.6 & 55.9 & 15.4 & 14.6 & 8.4 & 8.2 \\
\hline $45-49$ & 28.2 & 40.3 & 12.4 & 14.6 & 3.5 & 5.9 \\
\hline \multicolumn{7}{|l|}{ Promedio } \\
\hline Reportado & 58.8 & 57.4 & \multicolumn{2}{|c|}{$\%$ de Usuarias = } & 61.68 & 60.09 \\
\hline
\end{tabular}

$1986 \mathrm{Cc}=$ Índ. de anticoncepción $=1-(1.08 \times \mathrm{e} \times \mathrm{u})=0.4027$

(u) $=\%$ prevalencia $=61.68 ;(c)=0.8966$

$1990 \mathrm{Cc}=$ Ind. de anticoncepción $=1-(1.08 \times \mathrm{e} \times \mathrm{u})=0.4088$

$(u)=\%$ prevalencia $=60.09 ;(e)=0.9111$

\begin{tabular}{|c|c|c|c|c|c|}
\hline \multirow[t]{2}{*}{ método } & \multicolumn{2}{|c|}{$\begin{array}{l}\% \text { de usuarios } \\
\text { por método } \\
\text { (1) }\end{array}$} & \multirow{2}{*}{$\begin{array}{c}\text { Estandard de } \\
\text { efectividad } \\
\text { usuario } \\
\text { (2) }\end{array}$} & \multicolumn{2}{|c|}{$\begin{array}{c}\mathrm{e}(\mathrm{i}) \\
(\omega \times(1) \times(2))\end{array}$} \\
\hline & 1986 & 1990 & & 1986 & 1990 \\
\hline Esteriliza. & 35.0 & 42.7 & 1.00 & 0.21589 & 0.25656 \\
\hline Vasectomía & 1.2 & 1.7 & 1.00 & 0.00740 & 0.01021 \\
\hline Pastillas & 37.2 & 30.1 & 0.90 & 0.20651 & 0.16277 \\
\hline Inyecciones & 7.1 & 7.2 & 0.70 & 0.03066 & 0.03028 \\
\hline D.I.U. & 10.2 & 9.6 & 0.95 & 0.05977 & 0.05480 \\
\hline Esspumas & 0.9 & 0.7 & 0.70 & 0.00389 & 0.00294 \\
\hline Condón & 2.6 & 3.5 & 0.70 & 0.01123 & 0.01472 \\
\hline Diafragma & 0.3 & 0.3 & 0.70 & 0.00130 & 0.00126 \\
\hline Ritmo & 2.8 & 0.0 & 0.70 & 0.01209 & 0.00000 \\
\hline Retirg & 0.7 & 0.0 & 0.70 & 0.00302 & 0.00000 \\
\hline Oros & 0.3 & 3.3 & 0.70 & 0.00130 & 0.01388 \\
\hline N.E. & 1.6 & 1.1 & 0.00 & 0.00000 & 0.00000 \\
\hline Total & 100 & 100 & (e) $=$ SUMe(i) & $=0.8966$ & 0.9111 \\
\hline
\end{tabular}

FUENTE: CONEPO-BC, CRIM-UNAM, IIS-UABC,1987; IIS-UABC y CONEPO-BC,1993.

*: En 1990 "otros" incluye ritmo y retiro. 
CUADRO 8. B.C. población total, nativa y migrante: determinantes próximos de la fecundidad, índices de infecundabilidad (1986 y 1990).

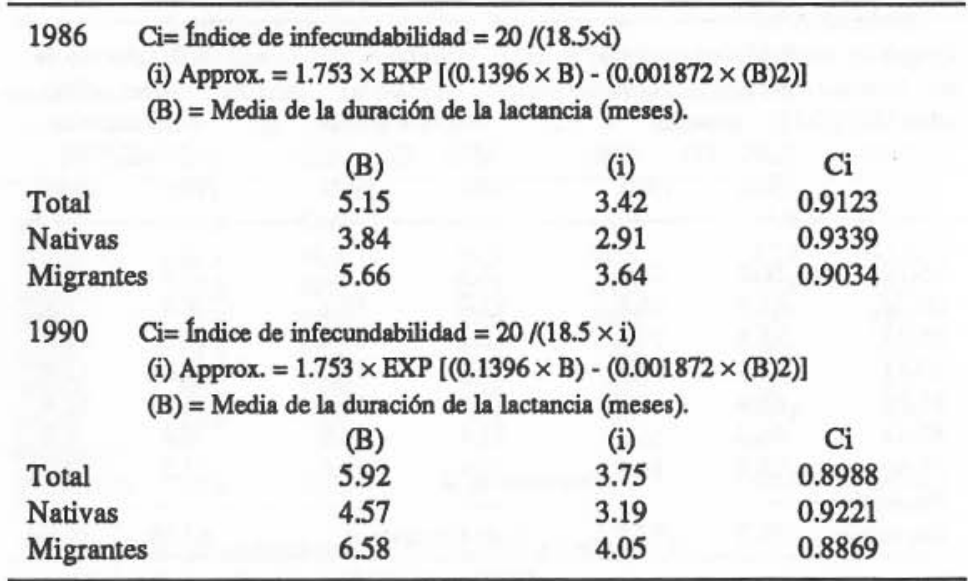

FUENTE: CONEPO-BC, CRIM-UNAM, IIS-UABC,1987; IIS-UABC Y CONEPO-BC, 1993.

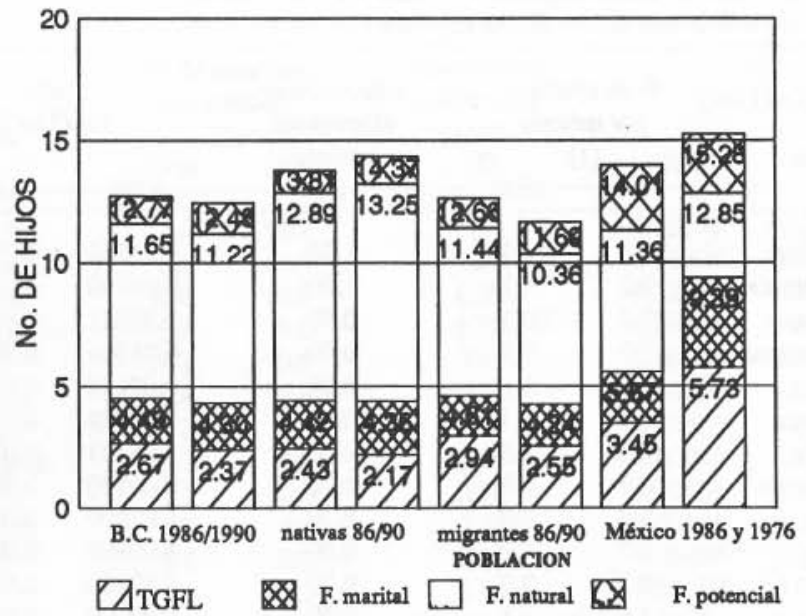

Figura 4. México y B.C. fecundidad potencial, natural, marital y TGFL (1976, 1986 y 1990).

Fuente: Cuadro 2. 
A partir de ese procedimiento, se logra establecer que las diferencias entre las TGFL de nativas y migrantes son de $21.0 \%$ y $17.5 \%$ en 1986 y 1990, respectivamente; que para esas mismas fechas, la contribución del índice de matrimonio $(\mathrm{Cm})$ a la diferencia es de $16.4 \%$ y $20.6 \%$; la del índice de anticoncepción (Cc) de $17.8 \%$ y $23.5 \%$; la del índice de infecundabilidad ( $\mathrm{Ci}$ ) de $-3.7 \%$ y $-4.2 \%$; en tanto que las diferencias en la fecundidad potencial (TF) contribuyen con $-9.6 \%$ y $-22.4 \%$, en 1986 y 1990 , respectivamente. En conjunto, estos resultados identifican al menos cuatro elementos fundamentales que sustentan las diferencias entre los patrones reproductivos de esos dos grupos.

El primer elemento, es el referido a la elevada contribución que generan los disímiles niveles de la fecundidad potencial (TF) de las migrantes y de las nativas (e.g. 11.7 y 14.4 en 1990, respectivamente) en las diferencias de las TGFL. Esa diferencia en los niveles de la TF, puede ser atribuida a variables intermedias no incluidas en nuestra aplicación del modelo de los determinantes próximos de la fecundidad; la más obvia, en consecuencia, sería la del recurso del aborto inducido.

Sin embargo, para que dicha variable pudiera explicar la diferencia en los niveles de TF, se requeriría que la prevalencia del aborto fuera superior entre las mujeres migrantes que entre las nativas, lo cual entraría en contradicción con los resultados de estudios comparativos internacionales que han encontrado que "la incidencia del aborto inducido generalmente se asocia positivamente con la residencia urbana y mayor escolaridad" (Casterline, et al., 1984:41). Es decir, que la incidencia del aborto se incrementa con las condiciones de vida y niveles de desarrollo que en nuestro caso corresponden de manera más próxima a la situación de las mujeres nativas.

Otra variable intermedia no incluida, y que se puede considerar para explicar las diferencias en los niveles de la TF entre nativas y migrantes, es la que atiende a la posibilidad de la separación temporal entre los cónyuges. Asumiendo que ésta fuese la causa de las diferencias en las TF, por ejemplo, se puede argumentar que con un plausible $8.33 \%$ de mujeres migrantes en unión no embarazadas con compafieros temporalmente ausentes, sería suficiente para reducir el valor de $\mathrm{Cm}$ de las migrantes para 1986 a 0.585 y con ello se igualarían los valores de TF.

El segundo elemento en que se sustentan las diferencias en los patrones reproductivos de las mujeres migrantes y nativas de Baja California, consiste en las crecientes aportaciones que generan los índices de infecundabilidad postparto por lactancia (Ci). Estas aportaciones -al igual que las referidas a la TF- muestran signos negativos tanto en 1986 como en 1990 , indicando que las mujeres migrantes reducen su fecundidad potencial 
de manera más pronunciada que las nativas a causa de sus prácticas de lactancia más prolongadas. Estas aportaciones de $\mathrm{Ci}$-que en principio dan muestras de una tendencia a incrementarse - aun para 1990 resultan relativamente menores, dado que constituyen menos del $5.0 \%$ de la diferencia en la TGFL de los dos grupos.

Por su parte, la aportación del tercer elemento a las diferencias en las TGFL de las migrantes y nativas se caracteriza por ser la más elevada, tanto en 1986 como en 1990. Se trata, en este caso, del índice de anticoncepción (Cc) que representa el $17.8 \%$ y $23.5 \%$ del desnivel de las TGFL en las dos fechas mencionadas. Este elemento, con sus elevados valores positivos y crecientes, muestra que la discrepancia en el patrón de anticoncepción de las migrantes y nativas es el determinante principal de los diferenciales de la fecundidad entre estos grupos, y que su importancia se ha incrementado en el último quinquenio de los ochentas, al generar mayores reducciones proporcionales en la fecundidad marital natural (TN) de las mujeres nativas que en la de las migrantes.

Finalmente, el cuarto elemento en que se sustenta la diferencia de fecundidad entre nativas y migrantes en Baja California, es el referido a los patrones de formación de uniones que caracterizan a cada uno de esos grupos. La aportación de este elemento a través del índice de matrimonio $(\mathrm{Cm})$ a esa diferencia, al igual que la del anterior, también ha sido positiva, elevada y creciente (i.e. $16.4 \%$ y $20.6 \%$ en 1986 y 1990). El signo de esa aportación, responde a la mayor reducción proporcional de la fecundidad marital (TM) de las nativas como consecuencia de que presentan menores proporciones de mujeres en unión que las migrantes en los siete grupos de edad considerados, en tanto que el incremento de sus valores entre 1986 y 1990 señala una diferenciación creciente en los patrones de formación de uniones de los grupos de mujeres migrantes y nativas de Baja California.

\section{CONCLUSIONES}

A partir de los resultados hasta aquí presentados, consideramos pertinente plantear tres líneas generales de conclusiones. La primera de éstas corresponde a las sugerencias metodológicas y de investigación que se derivan de este estudio. La segunda, por su parte, atiende al objetivo central de esta investigación, dado que aborda tanto los factores que explican los diferenciales de fecundidad entre migrantes y nativas de la entidad, como el aspecto cuantitativo de los efectos indirectos que la inmigración representa para la dinámica demográfica de Baja California. Por último, la tercera línea general de conclusiones se integra con la identificación de 
posibles vías de acción en materia de adecuación de la política demográfica a las condiciones específicas de esta zona del país.

En relación con los aspectos metodológicos y de investigación consideramos que, por una parte, los resultados obtenidos en este estudio permiten confirmar las potencialidades y bondades del marco conceptual de los determinantes próximos, al ser utilizado para propósitos de análisis en ei tópico de la relación entre migración y fecundidad. En este sentido, el hecho de que el modelo utilizado presente demandas relativamente bajas de información y de que a través de él se cuantifiquen los principales factores en que se sustentan los diferenciales de fecundidad en función de la movilidad de las poblaciones, lo califica como un recurso analítico en el que tal vez merezca más atención al estudiar la relación que nos ocupa.

Sin embargo, por otra parte, el caso que se ha estudiado también ha puesto de manifiesto que, para su cabal aplicación en el contexto de las EFNM, el modelo de los determinantes próximos de la fecundidad plantea demandas de información complementaria. Al respecto, baste reiterar que parte importante de la diferencia de fecundidad entre migrantes y nativas de Baja California, se puede atribuir a comportamientos y procesos probablemente contrastantes en materia de prácticas como el aborto inducido y la separación temporal de cónyuges (e.g. por migración laboral), y que estas prácticas y procesos deben formar parte del perfil reproductivo de la población fronteriza que - por vecindad- tiene acceso a servicios legales de aborto y a mercados internacionalizados de trabajo (Alegría, 1992). Estas peculiares condiciones plantean, pues, la necesidad de realizar esfuerzos para diseñar e implementar los instrumentos y métodos requeridos para completar la información que demanda el tópico de análisis sugerido.

La segunda línea general de conclusiones que derivamos de los resultados presentados, se refiere a los factores que explican los diferenciales de fecundidad entre migrantes y nativas, y al efecto indirecto que representa la inmigración para la dinámica poblacional de Baja California.

En relación a los primeros, por una parte, nuestro análisis basado en el modelo de los determinantes próximos de la fecundidad ha mostrado que el mayor nivel de fecundidad que se observa para 1990 en las mujeres migrantes en relación al de las nativas (i.e. TGF de 2.80 y 2.37 , respectivamente), se origina en que las primeras presentan mayores proporciones de nacimientos fuera de unión; mayores proporciones de mujeres en unión; menores proporciones de mujeres en unión que utilizan métodos de planificación familiar, y; que las diferencias de fecundidad se reducen marginalmente, debido a las duraciones más prolongadas en sus prácticas de lactancia postparto. 
Adicionalmente, por otra parte, el análisis comparativo entre 1986 y 1990 permitió establecer que las diferencias, en los cuatro elementos arriba mencionados, han tendido a acentuarse en el transcurso de ese lapso dando lugar, de esa forma, a la definición de dos patrones reproductivos crecientemente contrastantes entre las mujeres migrantes y nativas de la entidad.

Así, las diferencias que aquí se han mostrado en relación a esos patrones, plantean ahora la necesidad de avanzar la investigación hacia la identificación de los factores, variables o determinantes "remotos" del comportamiento reproductivo (e.g. niveles de escolaridad, participación en actividades económicas, niveles de ingreso, tipos de hogares, etc.), que aporten elementos de explicación en relación a los patrones observados de fecundidad ilegítima, formación de uniones, prevalencia de anticoncepción y prácticas de lactancia postparto de los dos grupos aquí abordados.

Cabe esperar que con la identificación de esos determinantes "remotos" de la fecundidad, se haga más amplia la gama de acciones a desarrollar para modular el impacto indirecto que la inmigración genera en la dinámica demográfica de la entidad. En relación a dicho impacto, se deben de tomar en cuenta al menos dos consideraciones.

En primer lugar se debe advertir que, utilizando las tasas especificas de fecundidad (TEF) incluidas en la sexta columna de los dos últimos paneles del cuadro 4 y las estimaciones derivadas de la encuesta de 1990 del número de mujeres migrantes y nativas para los siete grupos quinquenales de edad, ${ }^{8}$ sc obtiene un total de 43,247 nacimientos anuales que se integran por 20,005 de las nativas y 23,242 de las migrantes. La estimación total de nacimientos anuales, resulta muy similar al promedio de los nacimientos de menores de un año registrados en Baja California durante 1989 y 1990 (i.e. 43,836 : INEGI, 1992 b y 1993:16 y 11), en tanto que los nacimiento de mujeres migrantes sólo se reducen a 22,600 al utilizar las últimas cifras censales disponibles.?

Para contextualizar nuestras estimaciones de nacimientos, el segundo clemento a considerar es el relativo al hecho de que entre 1985 y 1990 , Baja California presentó un saldo neto migratorio positivo que equivale a aproximadamente 36,000 nuevos residentes por año durante ese lapso (INEGI, 1992a:18). Este dato permite advertir que, el efecto "indirecto" de

8 Las estimaciones de población femenina total de cada uno de los siete grupos de edad incluidos son, de menor a mayor: $76444,54308,40914,23681,14536,9755$ y 5912 para las nativas, y: $37453,46180,40391,42214,37231,33281$ y 27948 para las migrantes.

Se considera 1989 y 1990 dado que la encuesta de 1990 está centrada al 12 de marzo de 1990. Para derivar la población migrante del dato censal (nNEGI, 1992e:11), se utilizo la proporción de la muestra que representan estas mujeres en cada grupo quinquenal de edad (cuadro 4). 
la inmigración acumulada hacia Baja California (i.e. los 23,242 nacimientos correspondientes a las migrantes) representa el $64.6 \%$ del total anual de inmigrantes. Es decir, que el aporte total de la inmigración a la entidad (i.e. más de 59 mil nuevos habitantes anuales), se integra proporcionalmente de casi dos nacimientos (i.e. 1.94) por cada tres nuevos inmigrantes que arriban a la entidad por año.

Ante la magnitud de estos procesos, y dada la necesidad tanto de respetar el libre movimiento de la población dentro del territorio como de lograr que a nivel de la entidad se alcancen las metas poblacionales que se han fijado (i.e. crecimiento de $1.8 \%$ anual en 1994) para el país en su conjunto (S.G, 1991:19), el reto que se plantea consiste en definir e implementar líneas de acción en materia de política demográfica que respondan a las condiciones particulares de Baja California.

En ese sentido, los resultados aquí presentados dan pie a sugerir por lo menos tres líneas generales de acción. La primera consiste en orientar de manera prioritaria las acciones tendientes a elevar la edad a la primera unión, hacia los grupos de edad de mujeres migrantes actualmente adolescentes. A través de esta focalización se buscaría atenuar, en el mediano y largo plazo, las diferencias observadas entre nativas y migrantes en relación a los patrones de formación de uniones.

De igual forma, las actividades de los programas oficiales y no gubernamentales de planificación familiar, deberán estar dirigidas a lograr mayores niveles de aceptación en los grupos de mujeres migrantes unidas no-embarazadas, particularmente en el rango de edad de 15 a 25 años, dado que en estos últimos se han observado evidencias que indican que la prevalencia en el uso de anticonceptivos ha tendido a disminuir.

La tercer línea general de acción que se deriva de los resultados presentados, es la que corresponde a la necesidad de que a través de las instituciones de atención a la salud - tanto públicas como privadas- se promueva la práctica de la lactancia postparto y la prolongación de su duración, particularmente entre las mujeres nativas.

A partir de ese conjunto de actividades, que más que sustituir deben complementar a las que se dirigen a poblaciones-objetivo más amplias, se buscaría disminuir los periodos de exposición al riesgo de concebir y ampliar los periodos de infecundabilidad postparto e intergenésicos, con el propósito último de hacer compatibles los ritmos de crecimiento poblacional y los de desarrollo socioeconómico de Baja California. 


\section{BIBLIOGRAFÍA}

ALEGRÍA, T. 1992. Desarrollo urbano en la frontera México-Estados Unidos. Consejo Nacional para la Cultura y las Artes. Serie Regiones. México, D.F.

APPENDINI, K., Murayama, D. y Domínguez, R. 1972. "Desarrollo desigual en México, 1900 y 1960". En: Demografía y Economia, Vol.6, no.1. El Colegio de México. México, D.F.

BONGAARTS, J. 1978. “A Framework for Analyzing the Proximate Determinants ofFertility". En:Population and DevelopmentReview, Vol.4, no.1.

- 1982. "The fertility-inhibiting effects of the intermediate fertility variables". En: Studies in Family Planning, Vol.13, no. 6/7.

BONGAARTS, J. y Potter, R.G. 1983. Fertility Biology and Behaviour. An Analysis of the Proximate Determinants. Academic Press, N.Y.

BRAMBILA, C. 1985. Migración y formación familiar en México. El Colegio de México. México, D.F.

CABRERA, G. 1967. "La migración interna en México, 1950-1960". En: Demografia y Economia, Vol.1, no.3. El Colegio de México. México, D.F.

CASTERLINE, J., Singh, S., Cleland, J. y Ashurst, H. 1984. "The Proximate Determinants of Fertility". En: WFS Comparative Studies, No.39. ISI, Voorburg, Netherlands.

CONAPO. 1988. Demografia de la frontera norte de México. Consejo Nacional de Población. México, D.F.

CONEPO-BC, CRIM-UNAM, IIS-UABC. 1987. Encuesta demográfica de Baja California, 1986. Resultados. Mexicali, B.C.

CORONA, R. 1993. "Migración permanente interestatal e internacional, 1950-1990". En: Comercio Exterior, Vol. 43, no.8, agosto. Banco Nacional de Comercio Exterior. México, D.F.

CORONA, R. y Ruiz, C. 1982. "Notas sobre las rutas de migración interna en México". En: Investigación demográfica en México, 1980. CONACYT. México, D.F.

CORONA, R. 1991. "Principales características demográficas de la zona fronteriza del norte de México". En: Frontera Norte, Vol.3, no.5, ene-jun. El Colegio de la Frontera Norte. Tijuana, B.C.

CHÁVEZ, A. 1987. Migración, fecundidad y anticoncepción en Baja California (algunas hipótesis de trabajo). Universidad Nacional Autónoma de México. Centro Regional de Investigaciones Multidisciplinarias. México, D.F. 
DAVIS, Kingsley. \& Blake, Judith. 1956. "Social Structure and Fertility: an Analytic Framework". En: Economic Development and Cultural Change, no.4.

ESPINOZA, G. 1984. "Historia migratoria y fecundidad en la encuesta mexicana de fecundidad". En: Jiménez, R. y Minujin, A. (Coords.) Losfactores del cambio demográfico en México. Siglo XXI editores. México, D.F.

ESTRELLA, G. 1989. "Migraciones Interurbanas en la frontera norte de México". En: Estudios Fronterizos, vol. IX, no. 20. Instituto de Investigaciones Sociales de la UABC. Mexicali, Baja California.

FREJKA, T. y Atkin, L. 1990. "El aborto en América Latina". En: DEMOS. Carta demográfica sobre México. no.3.

GARCÍA Y GARMA, I. 1979. "Diferenciales de fecundidad en México, 1970". En: Demografia y Economia, Vol.13, no.1. El Colegio de México. México, D.F.

- 1989. "Estado del conocimiento relativo a los determinantes de los niveles de la fecundidad en México". En: Figueroa, B. (Ed.) $L a$ fecundidad en México; cambios y perspectivas. El Colegio de México. México, D.F.

HENRY, Louis. 1972. "On the measurement of human fertility". M.C. Sheps and E. Lapierre Adamyck (eds). Elsevier, Amsterdam.

INEGI. 1992a. Estados Unidos Mexicanos. Perfil sociodemográfico.XI censo general de población y vivienda, 1990. Instituto Nacional de Estadística, Geografía e Informática. Aguascalientes, Aguascalientes.

- $\quad 1992$ b. Estadisticas demográficas. Cuadernos de Población, No.3. Instituto Nacional de Estadística, Geografía e Informática. Aguascalientes, Ags.

- 1992c. Baja California. Resultados definitivos. Tabulados básicos.XI censo general de población y vivienda, 1990. Instituto Nacional de Estadística, Geografía e Informática. Aguascalientes, Ags.

- 1993. Estadisticas demográficas. Cuadernos de población No.4. Instituto Nacional de Estadística, Geografía e Informática. Aguascalientes, Ags.

IIS-UABC y CONEPO-BC. 1993. Encuesta demográfica de Baja California, 1990. Resultados. Mexicali, B.C. (en prensa).

MARGULIS, M. y Tuirán, R. 1986. Desarrollo y población en la frontera norte: el caso de Reynosa. El Colegio de México. México, D.F.

MONTERRUBIO, I., Flores, R. y Armada, M. 1993. "La transición demográfica de méxico por grupos de entidades federativas, a partir de sus tasas brutas de natalidad y mortalidad, 1940-1990". En: $I V$ 
Conferencia Latinoamericana de Población. La Transición Demográfica en América Latina y el Caribe. ABEP-CELADE-IUSSP-PROLAP-SOMEDE-INEGI-IISUNAM. México, D.F., vol. II.

MYERS, George C. \& Macisco, John J. Jr. 1975. "Revised Bibliography on Migration and Fertility". En: International Migration Review, vol.9.

NAG, M. 1980. "How Modernization can also Increase Fertility". En: Current Anthropology, vol. 21, no.5, octubre, The University of Chicago Press, Chicago, Ills.

NÚÑEZ, L. y Palma, Y. 1990. "El aborto en México". En: DEMOS. Carta Demográfica sobre México, no. 3

OCAMPO, E, 1981. "Consideraciones sobre la política de migración interna". En: Ocampo, E. et al. (eds.) Las migraciones y la poltitica demografíca regional en México. Asociación Mexicana de Población, México, D.F.

OJEDA, N. 1990. "Índices de masculinidad en tres ciudades fronterizas del norte de México: El mercado matrimonial en la región". En: Frontera Norte, vol. 2, no. 4, julio-diciembre, El Colegio de la Frontera Norte. Tijuana, B.C.

ORDORICA, M. 1976. "Migración interna en México, 1960-1970". En: Evaluación y Análisis, Serie III, No.5. Dirección General de Estadística, Secretaría de Industria y Comercio. México, D.F.

PAGE, H., Lesthaghe, R. y Shah, I. 1982. "Illustrative Analysis; breastfeeding in Pakistan". En: WFS Scientific Reports, no. 37. ISI, Voorburg, Netherlands.

QUILODRÁN, J, 1990. "Particularidades de la nupcialidad fronteriza". En: Estudios Demográficos y Urbanos, Vol. 5, no. 3, sep-dic. El Colegio de México. México, D.F.

SINGH, S., Casterline, B. y Cleland, J. 1985. "The Proximate Determinants of Fertility: Sub-national Variations". En: Population Studies, Vol.39.

S.G. 1991. "Programa Nacional de Población 1989-1994". En: Diario Oficial de la Federacion, 22-04-1991. Secretaría de Gobemación. México, D.F.

S.S.A. 1989. Encuesta Nacional sobre Fecundidad y Salud (ENFES). Secretaría de Salud y DHS. México, D.F.

S.P.P. 1979. Encuesta Mexicana de Fecundidad y Salud. Primer Informe de Resultados. Secretaría de Programación y Presupuesto, IIS. UNAM. México, D.F.

TABAH, L. y Cosío, M. 1970. "Medición de la migración interna a través de la información censal: el caso de México". En: Demografla y Economia, Vol. 4, no. 1. El Colegio de México. México, D.F. 
WARREN, Ch. 1992. "Determinants of Anglo and Mexican Fertility on the U.S.-Mexico Border". En: Weeks, J. \& Ham, R. (Eds.) Demographic Dynamics of the U.S.-Mexico Border. Texas Western Press-UTEP. El Paso.

WELTI, C. 1989. "La investigación del efecto de la anticoncepción sobre la fecundidad". En: Figueroa, B. (Ed.) La fecundidad en México; cambios y perspectivas. El Colegio de México. México, D.F.

ZENTENO, R. y Cruz, R. 1992. "A Geodemographic Definition of the Mexican Northem Border". En: Weeks, J. \& Ham, R. (Eds.) Demographic Dynamics of the U.S.-Mexico Border, Texas Western Press-UTEP, El Paso. 